\title{
Wetting and Dispersing Agents
}

\author{
Frank O.H. Pirrung ${ }^{a *}$, Peter H. Quednaua ${ }^{a}$, and Clemens Auschrab
}

\begin{abstract}
The incorporation of inorganic and organic particles into liquid resin media is an important process step in the paint, graphic arts and plastics industries. Various classes of wetting and dispersing agents are used for this purpose. The physical principles, chemistry and stabilisation mechanisms for both water- and solventbased applications are described. A focus is directed towards the molecular architecture of high molecular weight dispersants, which exhibit superior and more universal characteristics than low molecular weight wetting agents. A preview of future developments in this field completes this overview.
\end{abstract}

Keywords: Amphiphilic polymers · Colloid stabilisation · Dispersing agents · Living polymerisation · Pigment dispersion · Wetting agents

\section{Introduction}

Wetting and dispersing agents belong to the surfactant (surface active agent) family. Their design and characteristics are specially directed towards interactions at the solid-liquid interface between solid particles (such as pigments and extenders) and the liquid media in which they are to be stabilised. They should not be considered a member of the substratewetting group of surfactants which are used to improve the levelling of a liquid resin formulation or to prevent the formation of craters. The latter substances are described under the heading 'levelling agents'.

\section{The Dispersion Process}

The quality of a dry paint or ink film is strongly dependant on how finely and uniformly distributed are the solid parti- cles in the resin matrix. Defects like poor colour strength, insufficient covering power, low gloss and decreased weather resistance are typical examples known to the technician. Furthermore, a poorly dispersed system results in inferior mechanical properties of the dry film [1][2].

To obtain a finely dispersed system, the solid-liquid interface has to be modified during the grinding step in the production of paint or ink. During grinding, the pigment agglomerates and aggregates are crushed to primary particles and the newly formed surfaces are wetted by the liquid carrier (a solvent- or water-based resin solution) [3]. The use of a wetting agent can increase the speed at which the liquid phase (organic or aqueous) wets the pigment surface. This is important as the grinding step is the most time- and energy-consuming phase during the paint and ink production process. Another important consideration is the consecutive stabilisation of the fresh dispersion to prevent flocculation of the particles (Scheme 1). Flocculation can cause colour shift, sedimentation of the pigment particles and changes in viscosity of the dispersion.

The origin of flocculation is found in the Brownian motion of particles [1]. Small particles (here typically $0.01-1 \mu \mathrm{m}$ ) move randomly in the suspension, collide with each other and form flocculates, reducing their total surface energy. The smaller the particles, the stronger the Brownian motion. If, in addition to being smaller, their concentration is also high, the probability of encounter is increased and the tendency to flocculation is increased. In modern colour design, with the use of organic pigments (very small particles), this effect is more pronounced than in traditional systems with mainly inorganic pigments (large particles). This makes the use of additives obligatory.
${ }^{\star}$ Correspondence: Dr. F.O.H. Pirrunga

Tel.: + 31513657500

Fax: + 31513657550

E-Mail: efka@efka.com

aEFKA Additives BV

Innovatielaan 11

NL-8466 SN Nijehaske

${ }^{\mathrm{b}}$ Ciba Specialty Chemicals Inc.

Schwarzwaldallee 215

CH-4002 Basel

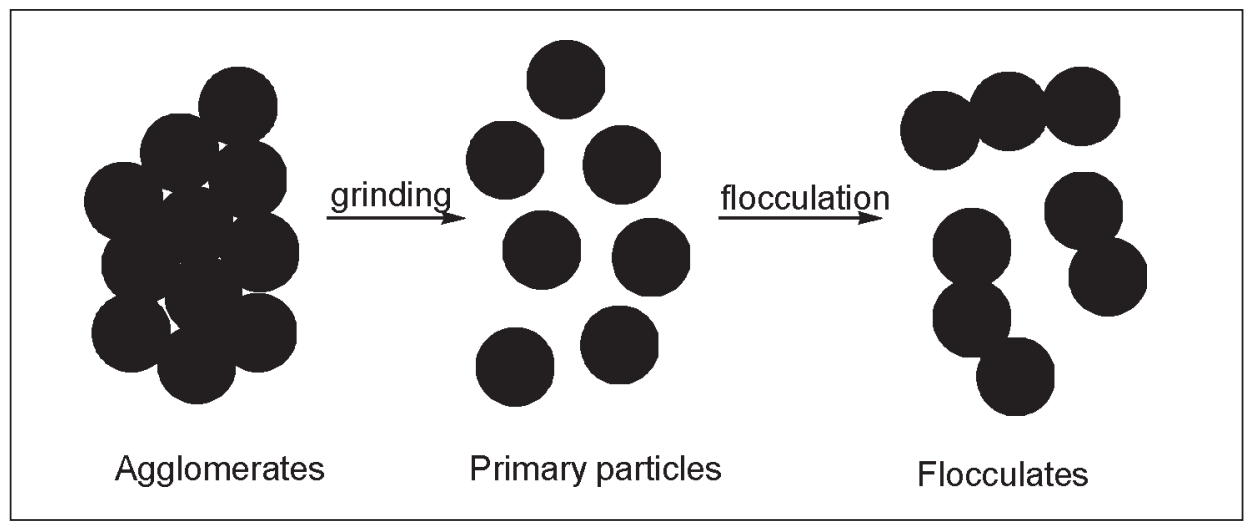

Scheme 1. Pigment grinding and flocculation 


\section{Stabilisation Mechanisms}

Scientifically, two main stabilisation mechanisms can be distinguished: electrostatic and steric stabilisation.

Electrostatic stabilisation takes place when particles bear the same electrical surface charge and as a result, repulsion takes place. The principle is based upon the DVLO theory [4]. The charge around the particle is organised into a double layer in which each layer possesses equal charge. When two particles approach one another their charged double layers overlap and repulsion takes place. At the same time, London-van-der-Waals forces lead to attraction of the particles. If the attractive forces are stronger than the repulsive forces, the dispersion will be unstable. However, if repulsive forces predominate, the system will be non-flocculating (Fig. 1). As this mechanism relies on the separation of ionic charges, it is mainly relevant in systems of high polarity like aqueous paints.

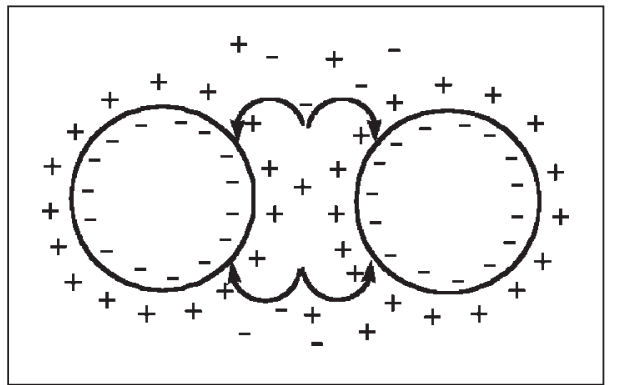

Fig. 1. Electrostatic stabilisation
Steric stabilisation is brought about by adsorbed polymers and applies to both water- and solvent-based systems [5]. This stabilisation is dependant on the structure and the dimensions of the adsorbed polymer layer. The polymer can adsorb onto a particle through the socalled 'anchoring groups', or segments that have strong affinity for the chemistry of the surface. The remainder of the polymer can be seen as 'dissolved' and can extend into the resin medium. These extended parts of the stabilising polymer become the first contacts between two approaching particles. A certain degree of inter-penetration between the polymer layers from the adjacent pigment particles is possible. As the concentration of solvated chains increases with decreasing distance, the free energy of the system increases. As a result of osmotic pressure, the solvent molecules start to migrate into the zone of the interpenetrating polymer layers and re-separate the two particles until a lower energy level is reached. In this way equilibrium is attained between penetration and repulsion (Fig. 2). The diameter of the adsorbed layer determines whether the distance between the particles is large enough to overcome the van-der-Waals attraction forces between them. In practice it is generally accepted that a molecular weight of at least $5000 \mathrm{~g} / \mathrm{mol}$ is required to achieve sufficient distance ( $100 \AA$ is considered to be optimal for most particles) [1][6].

The statements above make it clear that the non-adsorbed parts of the polymer must be adequately soluble in the liquid phase, otherwise the stabilising layer will collapse with immediate flocculation as a result. This is an important criterion
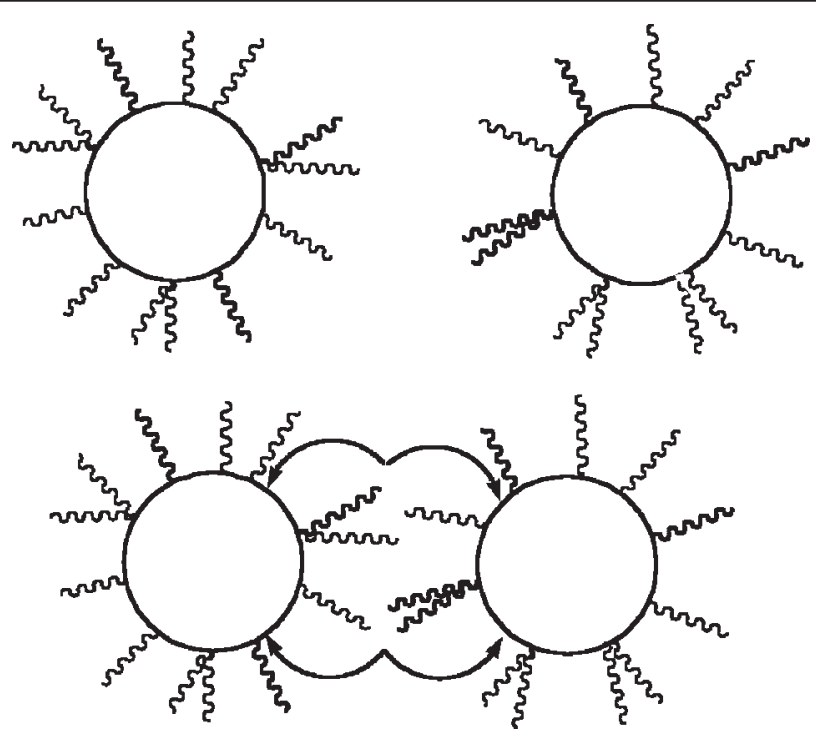

Fig. 2. Steric stabilisation for the selection of a dispersing agent for a given resin system; the solvent or carrier molecules must be able to migrate easily into the penetrating layers. Likewise the compatibility of the dispersant with the resin molecules will determine the effectiveness of the dispersant.

In general, effective steric stabilisation has to fulfil several conditions [2][6]:

- the particle surface has to be totally covered with dispersant,

- the layer has to be of sufficient diameter,

- the polymer has to be strongly adsorbed on the surface to avoid desorption upon collision or dilution of the dispersion,

- the steric chains should be linear and extend into the medium to build up a protective layer.

\section{Wetting and Dispersing Additives}

In day-to-day practice, the paint and ink industries employ low molecular weight $(<2000 \mathrm{~g} / \mathrm{mol})$ as well as high molecular weight $(5000-25000 \mathrm{~g} / \mathrm{mol})$ wetting and dispersing agents.

\subsection{Aqueous Systems}

Low molecular weight structures are called wetting agents and are mainly focused upon applications in water-based systems. Their purpose is to wet the surface of the particles that are being incorporated. This maintains the concentration equilibrium in the system of other surfactants that are needed to stabilise the resin emulsion. This prevents them from orienting themselves towards the surface of the pigment causing a reduction in the solubility of the resin leading to undesired viscosity increase. Classical pigment wetting agents used for this purpose are alkylphenol ethoxylates, which however have recently come under suspicion, and are more and more substituted by APE-free compounds (Fig. 3) [7].

The solid particle can be characterised by its zeta-potential, which shows the degree to which electrostatic stabilisation is possible. The purpose of dispersing agents in aqueous media is mainly to shift the (net) zeta-potential at the particle/liquid interface to a negative value in order to eliminate the attractive forces. By increasing the charge on the surface, approaching particles experience a stronger repulsion. This form of electrostatic stabilisation is very efficient for systems consisting of only one type of pigment with the same charge. 


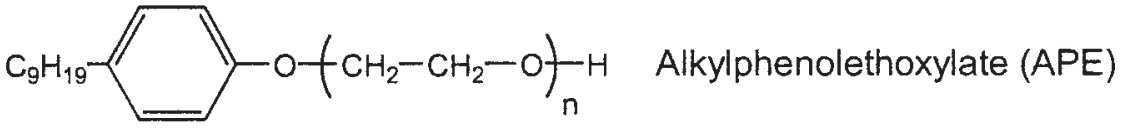

$\left.\mathrm{C}_{13} \mathrm{H}_{27}-\mathrm{O}+\mathrm{CH}_{2}-\mathrm{CH}_{2}-\mathrm{O}\right)_{\mathrm{n}} \mathrm{H}$

Alkylethoxylate

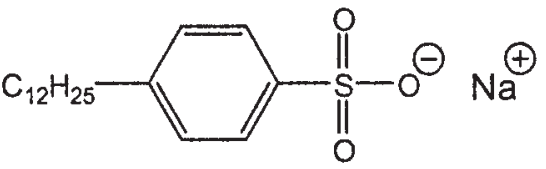

\section{Alkylbenzylsulfonate}<smiles>C[N+](C)(C)[13CH3]</smiles>

Fig. 3. Examples of low molecular weight wetting agents

Examples of inexpensive compounds that act on charge-repulsion are polyphosphates or polyacrylates (Fig. 4) [8].

Both systems are neutralised or partly neutralised to achieve water-solubility, and bear a negative charge that increases the total surface charge of the particle. Polyacrylates are considered to be more modern systems as they are less environmentally harmful.

Due to the fact that all pigments and extenders carry a different charge it is practically impossible to stabilise combinations of different pigments by this method. For combinations of pigments, it is necessary to use polymers that can - in addition to providing an electrostatic effect - also build up a steric stabilisation around the pigment particles to avoid the flocculation of the particles. Compounds that can build up steric hindrance are mainly based on polymers made through radical polymerisation techniques, such as dispersants with a styrene/maleic an- hydride (SMA) backbone, or acrylic copolymers. Both can be made water-soluble either by neutralisation or optional carboxylic side groups. A drawback of neutralised polymers is that they undergo partial protonation of carboxylic groups at $\mathrm{pH}$ values lower than 8 . This causes the polymer to lose some of its water solubility. Alternatively dispersant polymers can be directly obtained as water-soluble (or emulsifyable in aqueous media) by the incorporation of sufficiently polar side chains such as polyethylene glycol, polypropylene glycol or other moieties. The advantage of the latter approach is that the dispersant polymers are usable over a wide range of $\mathrm{pH}$ values, i.e. $\mathrm{pH} 4-11$. All polymers have in common that they have hydrolysable ester-groups in their structures, which saponify at very low $(<4)$ or very high $(>11) \mathrm{pH}$ values.

A special group of wetting agents for aqueous systems are products to enhance colour acceptance of universal tinters/

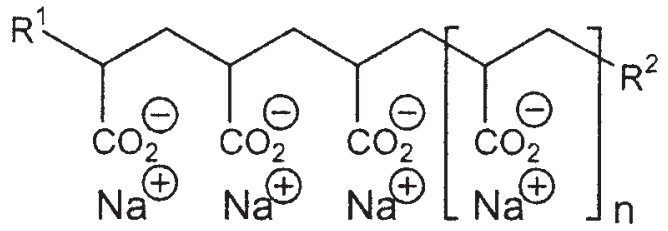

Polyacrylate

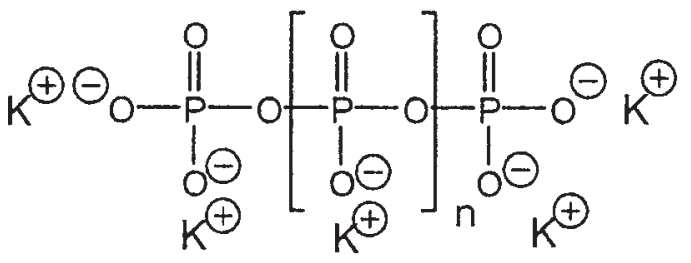

Polyphosphate colorants in aqueous and solvent-based base coats. Universal colorants are characterised by their high polarity and high loading of wetting agents for dispersion of pigments. The carrier of universal colorants is water and/or polyglycols. Upon addition of these pigment pastes to the base coats, the concentration difference of the surfactants in the system causes migration of the wetting agents from the colour paste into the bulk, leading to seeding, loss of colour strength and flocculation of the pigments. The special wetting agents for colorants are for example surfactant-like molecules with pigment affinic groups and can be used in two ways: they can be added to the base coat to compensate the concentration gradient of wetting agents after addition of the colorant, or can be used directly in the colour paste if they are designed to have a better pigment affinity and therefore less tendency to migrate into the bulk.

\subsection{Solvent-based Systems}

Likewise in organic solvent-based systems, both low and high molecular weight dispersants are in use. In principle, the dielectric constant of most organic solvents - though not completely negligible - is low. This prevents efficient electrostatic repulsion. Consequently stabilisation mechanisms and the mode of action of dispersants in organic media are based on different principles.

a) Monofunctional wetting agents are used to wet and disperse pigment particles, especially the inorganic types. A hydrated layer surrounds the inorganic pigment particles and - if faster dispersion is to be achieved - this hydrophilic surface must be made compatible with the hydrophobic synthetic binder solution. Here the low molecular wetting and dispersing agents can act as a bridging agent and result in a strong viscosity reduction allowing higher pigment loads and a faster grinding step (Scheme 2).

b) In primers and fillers multifunctional wetting and dispersing agents help to obtain a high loading grade of inorganic particles. They are equipped with several hydroxyl or carboxylic acid groups and, after the grinding step, are able to build up a lattice structure with inorganic particles in the liquid medium. This counteracts the tendency of these inorganic particles - facilitated by their high density - to sedimentation. Secondarily, multifunctional wetting and dispersing agents provide anti-sagging behaviour to the wet paint upon applica-

Fig. 4. Polymers for electrostatic stabilisation 


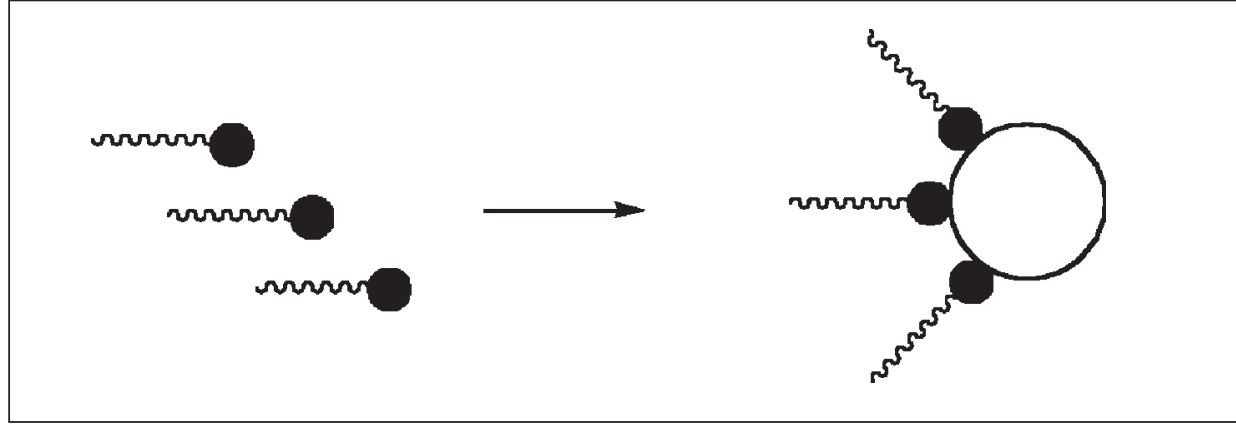

Scheme 2. Monofunctional wetting and dispersing agents tion on vertical substrates. Similar anti-sagging behaviour can be obtained by use of rheological modifiers - but without the primary pigment wetting advantages offered by multifunctional wetting and dispersing agents (Scheme 3 ).

c) Intentional re-flocculation of the inorganic pigments in co-grinding of organic and inorganic pigments is also achieved with multifunctional wetting agents. In such systems generally the organic particles are less stabilised and this leads to flooding and floating effects of the inorganic pigment. This intentional re-flocculation of the inorganic pigments leads to a reduced mobility to avoid local floating and flooding effects such as Bénard cells during the drying process and storage. Flooding is defined as a homogenous change of colour at the whole surface of the wet film, and can be easily detected by performing a rub-out test [8].

The introduction of high molecular wetting and dispersing agents (HMWD) follows from the growth of modern topcoats, in conjunction with an increasing use of high-performance organic pigments and mixtures thereof. This applies to both industrial (e.g. automotive) and decorative applications.

To understand the specific advantages of HMWDs, it has to be explained that organic pigments are more difficult to wet and stabilise due firstly to their small primary particle size, which gives a larger total surface to be wetted. Secondly, organic crystal structures do not, in general, have uniform surfaces. On a single crystal, faces of high and low polarities can be distinguished. Thirdly, due to the small particles, Brownian motion has more influence on flocculation; as the low molecular weight dispersant is constantly wetting and de-wetting the pigment surface, the Brownian contribution to flocculation is more difficult to control and additional steric stabilisation becomes necessary.

Lastly, the surfaces of organic pigments have fewer (or no) ionic groups compared to inorganic particles, so that interactions with the resin molecules and the low molecular weight polymers used in the paint and ink industry are much weaker. Organic pigments contain mainly chromophoric groups consisting of $\mathrm{C}$, $\mathrm{H}, \mathrm{O}$ and $\mathrm{N}$ atoms that are usually conjugated and bear optional substituents [10]. The pigment producer tries to enhance

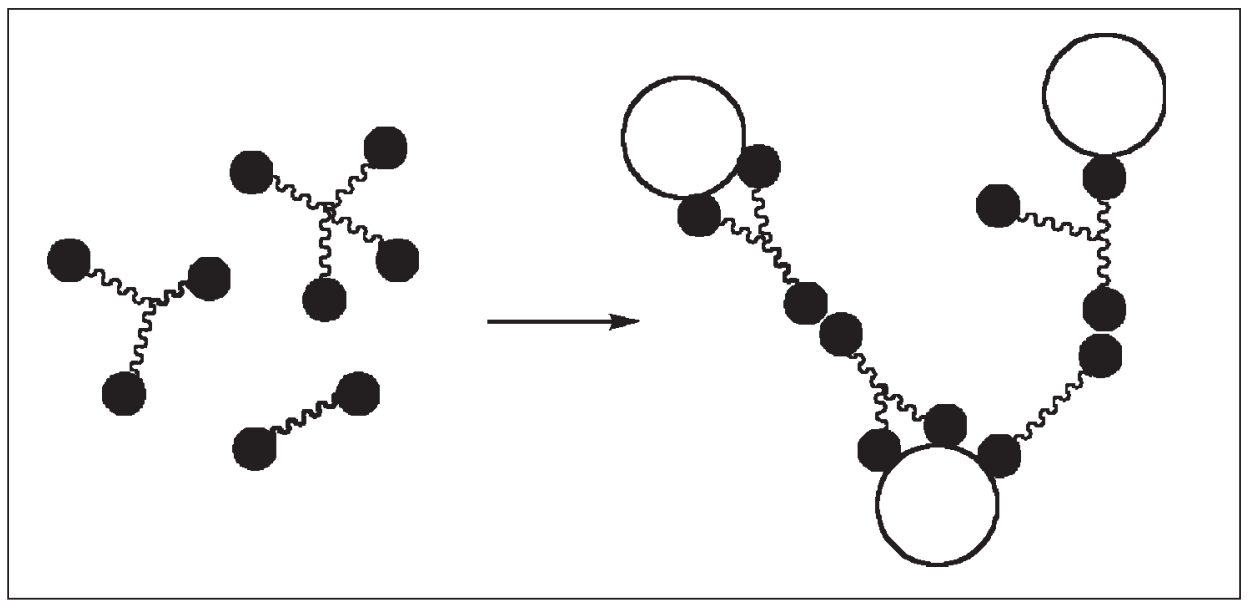

Scheme 3. Multifunctional wetting and dispersing agents the wettability of his pigments by posttreatment of the surface with polar pigment derivatives or other organic mainly poly-cyclic moieties carrying one or several acid or amino groups. Aminic groups combined with aromatic rings have proven to be effective anchoring groups for high molecular weight dispersants, as these moieties can readily form hydrogen bridges, dipole-dipole interactions and van-der-Waals forces (listed in decreasing strength) between the dispersant and the organic pigment surface. Due to the fact that these forces lead to a much weaker adsorption than the ionic forces that predominate on inorganic surfaces, dispersants for organic particles must contain a variety of anchoring groups (on each molecule), which can be distributed blockwise or statistically over the polymeric chain to ensure complete adsorption of the polymer. It has been proven that these anchoring groups also function on inorganic surfaces of pigments and extenders, since these anchoring groups can be polarised so that they also interact with the charged surface.

Another important aspect of these dispersants is their high molecular weight. High molecular weight molecules are important not only to accommodate all the necessary anchoring groups, but also to ensure sufficient distance in space between the particles in the dispersed stage. However, there are practical limits on the molecular weight of the dispersant since paint and ink formulations are liquid media and all components of this solution have to show sufficient solubility. Another factor which limits molecular weight is the formation of bridges between particles or with the resin molecules, leading to undesired increase of viscosity and formation of structure. From experience, the best results are obtained with molecular weights between 5000 and $25000 \mathrm{~g} / \mathrm{mol}$. The minimum requirement for steric stabilisation to occur lies around $5000 \mathrm{~g} / \mathrm{mol}$.

\section{Chemical Architecture of High Molecular Weight Dispersants}

As previously mentioned, high molecular weight dispersants are gaining in importance in modern pigment dispersion technology, due to the growing demand for organic pigments with small particle sizes and difficult-to-wet surfaces. For high molecular weight dispersants the stabilisation of the finely dispersed particles is quite a challenge. Consequently efficient anchoring mechanisms are required. The state of the art shows 


\begin{tabular}{|c|c|}
\hline Random (linear) & ABACDBBACA... \\
\hline Block (linear) & AAAAABBBBBB... \\
\hline Tapered (linear) & AAABBBCCCCCAAABB.. \\
\hline Hybrid & ABBCACA-XXYYYXZ... \\
\hline Random (branched) & 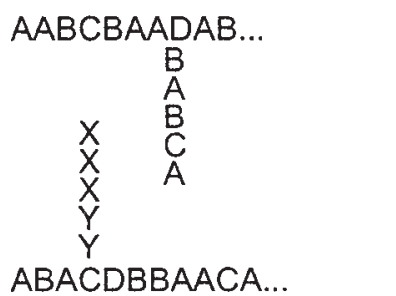 \\
\hline
\end{tabular}

(A, B, C, D, X, Y, Z = monomeric units)

Fig. 5. Polymeric architecture

that a series of polymeric architectures have proven to be suitable for this purpose (Fig. 5):

- random copolymers: polyacrylates and modified styrene-maleic anhydride copolymers,

- block copolymers: polyurethanes, polyesters and polyacrylates,

- tapered copolymers: polyacrylates,

- hybrid structures: polyurethanes and polyesters.

These polymers can be linear, branched or grafted.

All these architectures have the common feature that they are composed of defined regions of functionality. These can be identified as the backbone of the polymer carrying a) pigment affinic groups, b) steric side chains and c) groups to improve the compatibility with the resin system (Fig. 6). A further conformity is the molecular weight range of these polymers, which is generally inbetween 5000 to $25000 \mathrm{~g} / \mathrm{mol}$.

From the patent literature, a wide range of anchoring moieties are mentioned, which can be classified into the following categories [11]:

- Acidic anchoring groups: $-\mathrm{CO}_{2} \mathrm{H}$, $-\mathrm{SO}_{3} \mathrm{H},-\mathrm{PO}_{3} \mathrm{H}_{2}$, including their neutralised forms.

- Electroneutral groups: polyether, aromatic rings (substituted phenyl and naphtyl moieties) and heterocyclic derivatives thereof.

- Aminic anchoring groups: aniline, indole, imidazole, imide, morpholine, oxazolines, piperazine, polyethyleneimine, pyridine, trialkylamines, triazole can be considered as typical ex-

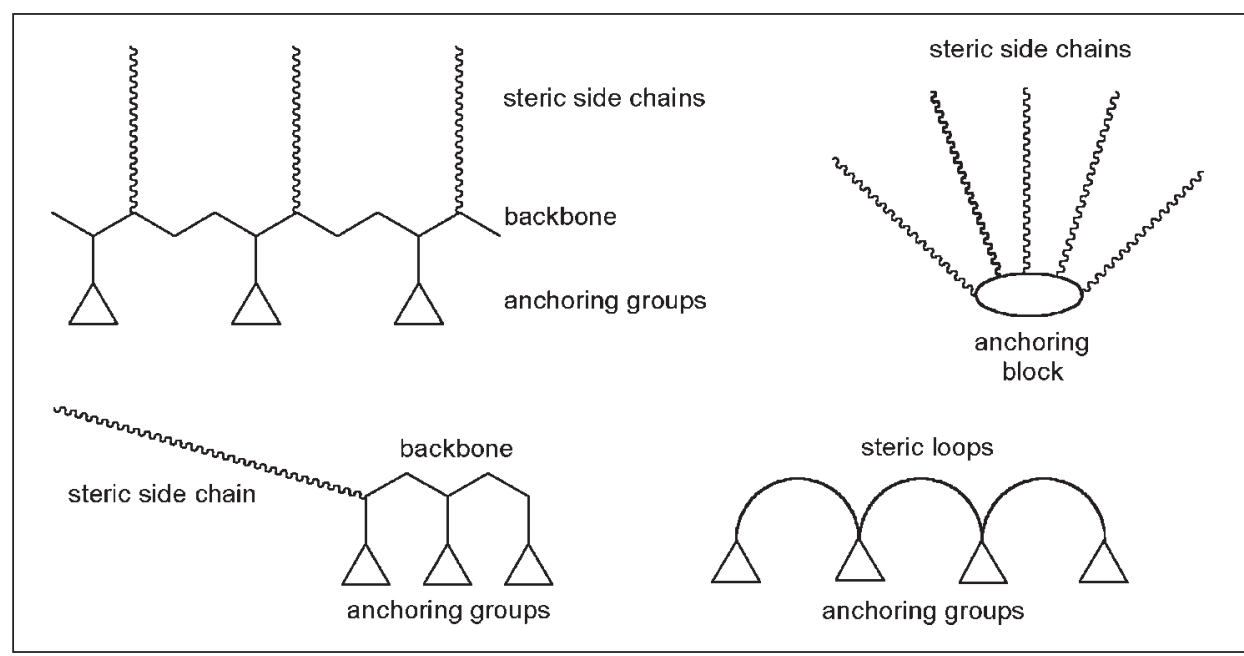

Fig. 6. Polymeric dispersant design amples. In special cases, the quaternised amine structures have given good results in pigment adsorption.

All functional moieties mentioned above are chemically connected through spacers to the backbone of the polymeric dispersant, where they are attached through appropriate reactive groups, like ethylenically unsaturated bonds, hydroxy, amino, epoxy, isocyanate or others.

In order to obtain dispersants with a wide application range with regard to pigment type, combinations of the previously mentioned anchoring categories can be used. For best results practice has shown that several anchoring groups per polymer molecule should be incorporated to achieve stable adsorption on the pigment surface.

For practical reasons, the steric side chains and the compatibility enhancing groups cannot be absolutely distinguished. For this purpose, polyesters based on condensates of fatty acids and fatty alcohols with hydroxy carboxylic acids and lactones yielding monohydroxy- or monocarboxy-functional linear chains can be attached to the polymer backbone. Also polyethylene and polypropylene glycol containing esters are used for build-up of steric hindrance.

In the case of polyacrylate dispersants, the non-functionalised part of the acrylic copolymer chain can act as a steric barrier. With random and tapered copolymers this can be visualised in the form of extending loops, while block copolymers can stretch a linear or branched acrylic chain into the medium (Fig. 6).

Polar polymers play the dominant role for aqueous based systems. The number of polar side chains (i.e. polyether chains) on the polymer backbone determines whether the dispersant is emulsifyable or totally soluble in water. Additionally water compatibility can be achieved by deprotonation of free carboxylic acid groups on the polymer backbone by amines or caustic solutions.

\section{New Approaches and Future Developments in Polymeric Pigment Dispersants}

In the coatings industry, environmental issues like the replacement of heavymetal based pigments and the trend to high-solids coatings, as well as economic aspects, continue to increase the demands on modern pigment dispersants. Such trends in coatings as well as demands from other important application fields like ink jet technology have stimulated 
intensive research for new polymeric pigment dispersants in recent years.

Research efforts have been directed towards improved control on polymer architecture by new techniques of 'living polymerisation'. A 'living polymerisation' is characterised by the fulfilment of certain kinetic criteria of the polymerisation reaction and the absence of polymer chain terminating side reactions [12]. This 'living character' allows precise control on molecular weight and distribution and enables the synthesis of block copolymers by sequential polymerisation of different monomers. Block copolymers are believed to belong to the most efficient structures of pigment dispersants [13].

The pioneering work of Webster et al. on group transfer polymerisation (GTP) has opened the path for the living polymerisation of a range of vinylic monomers [14]. This technique is used intensively for the synthesis of methacrylic block copolymer dispersants for applications in paints and ink jet applications [15]. Despite powerful control on polymer architecture, GTP has not found widespread industrial use. Disadvantages of the method are the stringent requirements on the purity of the reactants and limited availability and difficult handling of the reactive silylketene acetal initiators. Also the range of functional monomers that can be used, is relatively limited to monomers without acidic hydrogen or special monomers with protected groups.

In recent years promising new techniques for 'controlled free radical polymerisation' (CFRP) of vinylic monomers have been developed. The most prominent methods are 'atom transfer radical polymerisation' (ATRP) [16] and nitroxyl-mediated controlled free radical polymerisation (NOR) [17]. Both techniques allow the 'living' polymerisation of a broad range of acrylic monomers under conditions that are attractive for industrial use. The basic working principle of these techniques of CFRP is similar. A growing polymer chain with its active radical chain end is reversibly capped by a terminating species. The very low concentration of active growing polymer chains suppresses unwanted side reactions and creates a 'living' polymer chain, which can further grow by monomer addition. In case of ATRP a halogenterminated polymer chain is reversibly activated by a $\mathrm{Cu}$ (I) complex. In case of NOR-mediated CFRP, a stable nitroxyl radical reversibly caps the growing polymer chain (Scheme 4).

ATRP has been used for the synthesis of new block copolymer type and comb copolymer type pigment dispersants [18]. Scheme 5 shows an example of the synthesis of an acrylic block copolymer pigment dispersants with aminic anchor groups by ATRP.

Only recently new NOR polymerisation regulators have been developed which are able to affect controlled radical polymerisation of a wide range of acrylic monomers [19]. This broadens the basis
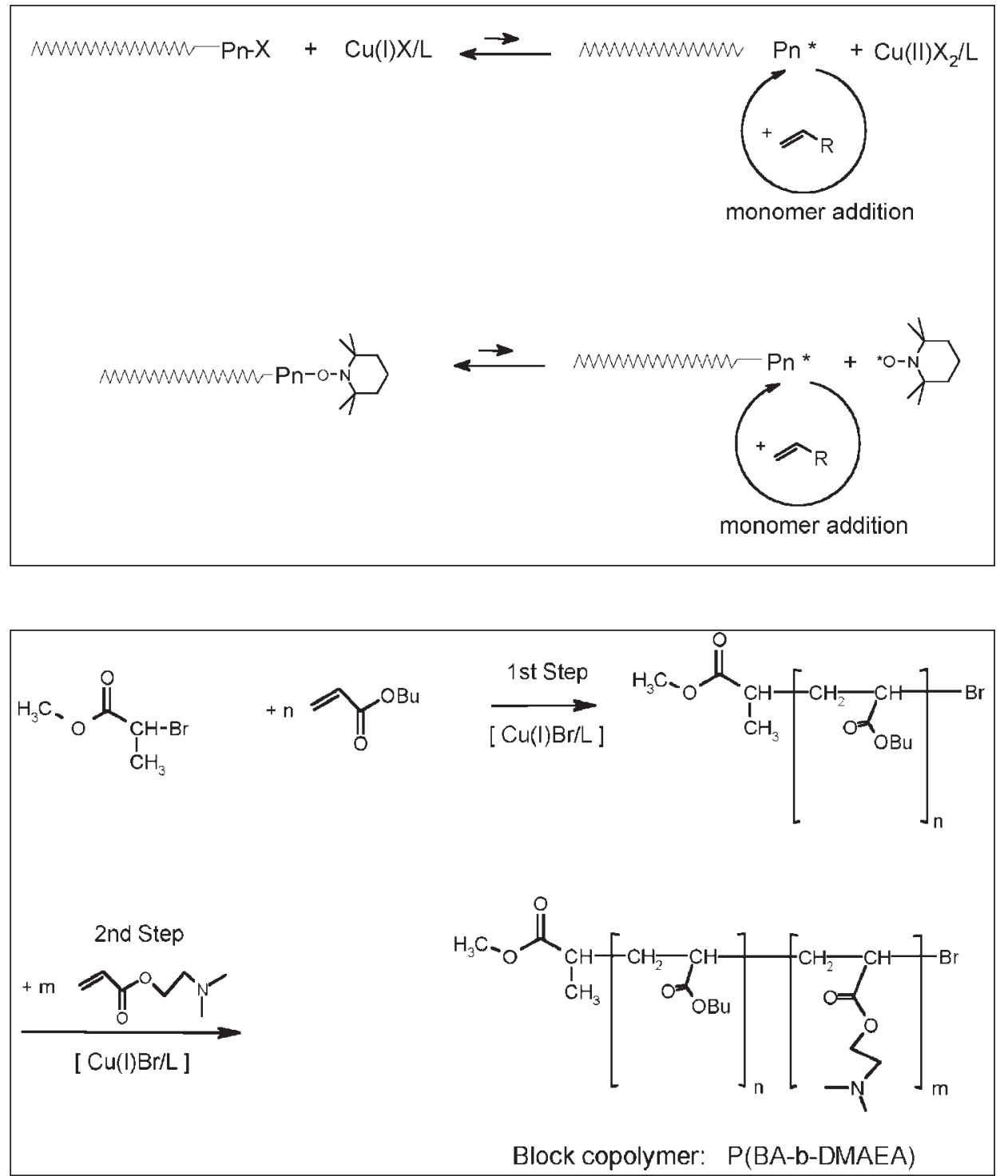

Scheme 4. Mechanism of controlled free radical polymerisation $(X=$ halogen, $L=$ ligand, $\mathrm{Pn}=$ polymer chain with $\mathrm{n}$ repeat units)

Scheme 5. Synthesis of block copolymer dispersants $\mathrm{P}[\mathrm{Ba}-\mathrm{b}-\mathrm{DMAEA}]$ by ATRP [18] 


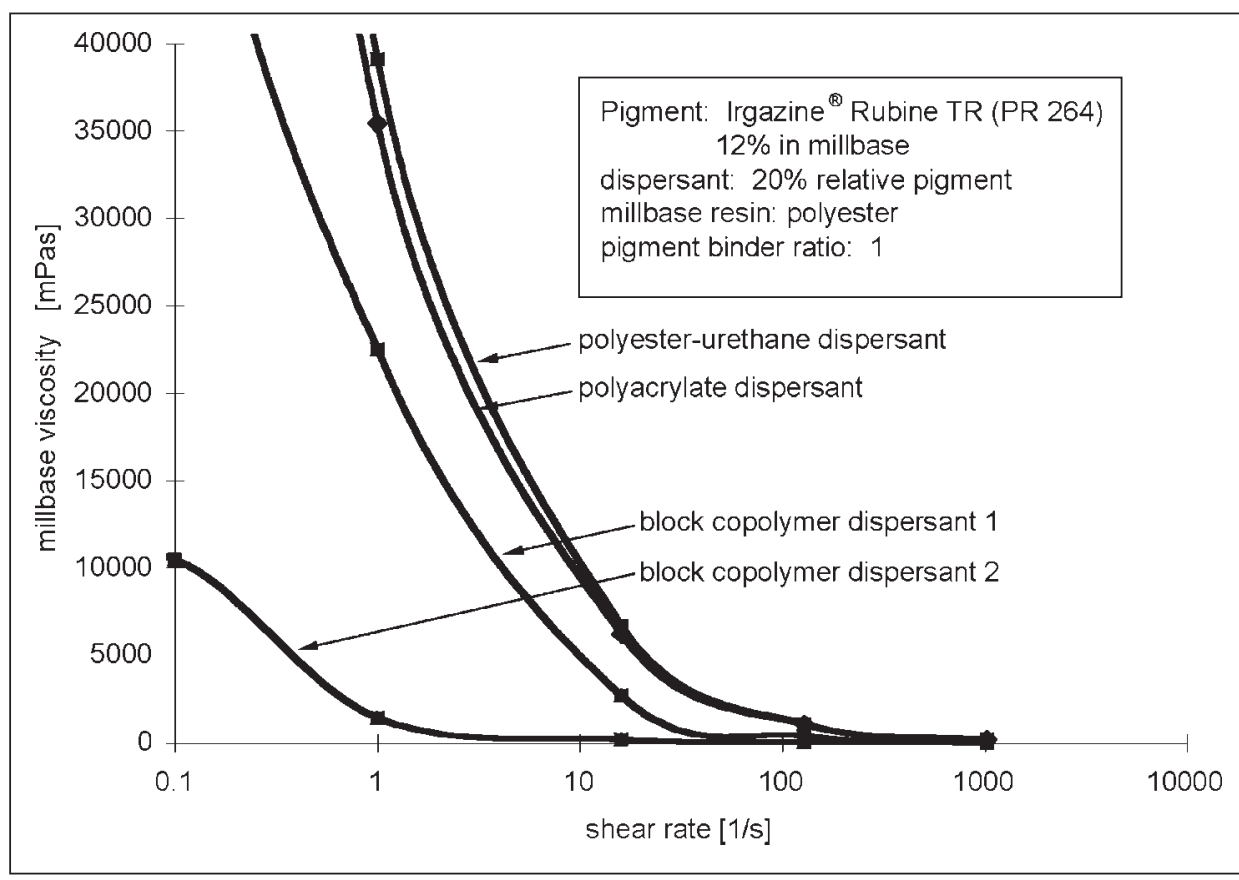

Fig. 7. Rheological performance of block copolymer dispersants in a millibase of a transparent organic pigment. Block copolymers 1 and 2 are acrylic block copolymers with different pigment affinic groups.

for future developments of pigment dispersants by CFRP. Fig 7 shows an example of the superior application performance of acrylic block copolymer dispersants made by controlled free radical polymerisation.

This example demonstrates that the viscosity of pigment concentrates can be well controlled by properly selected block copolymer dispersants. The performance with respect to specific pigments can be optimised by changing the pigment affinic groups. Such tailor-made dispersants allow for the use of higher pigment loads in the grinding step and offer improved flocculation stability in paint formulations.

\section{Conclusion}

Various classes of wetting and dispersing agents are used industrially for the colloidal stabilisation of pigments in different applications. For most applications, properly selected high molecular weight polymeric pigment dispersants offer superior performance. Polymeric pigment dispersants allow for the efficient manufacture of pigment dispersions and enable the formulator to make full use of the colouristic properties of pigments in their end use.

The main molecular design parameters, which determine the performance in a given application, are the chemistry and architecture of polymeric backbone, pigment affinic groups and steric stabiliser chains as well as overall molecular weight.

New innovative methods for controlled free radical polymerisation have paved the way to realise polymer architectures with a much higher degree of structural control. These new synthetic methods, combined with thorough understanding of the interactions of the functional polymers with pigments and coating materials, form the basis for the development of new dispersants with tailor-made properties. Promising further developments can be expected in the near future based on these living polymerisation techniques and will help to meet the increasing technical demands of modern coating systems.

Received: March 28, 2002

[1] a) J.H. Bieleman, in 'Lackharze', Ed. D. Stoye, W. Freitag, Carl Hanser Verlag, 1996, p. 647; b) J.H. Bieleman, in 'Lackadditive', Ed. J.H. Bieleman, Wiley-VCH Verlag GmbH, Weinheim, 1998, p. 67.

[2] J. Reck, L. Dulog, Farbe + Lack 1993, 99, 95.

[3] J. Schröder, Farbe + Lack 1985, 91, 11.

[4] E.J. Verwey, J.T.G. Overbeek, 'Theory of Stability of Lyophobic Colloids', Elsevier, Amsterdam, 1948
[5] D.H. Napper, 'Polymeric Stabilisation of Colloidal Dispersions', Academic Press, London, 1983.

[6] R. Jerome, Farbe + Lack 1992, 98, 325.

[7] P. Nanetti, 'Lackrohstoffkunde', Vincentz Verlag, Hannover, 1997.

[8] R. Hond, Verfkroniek 1994, 6, 44.

[9] W. Schulze, R. Werle, 'DispersionsSilikatsysteme', Expert Verlag TAW, Esslingen, 1995, p. 378.

[10] W. Herbst, K. Hunger, 'Industrial Organic Pigments' VCH Verlagsgesellschaft mbH, Weinheim, 1997.

[11] a) W.A. Wulff, P.H. Quednau (EFKA Additives), EP 311157-B1, 1987; b) P.H. Quednau, F.O.H. Pirrung (EFKA Additives), USP 5,882,393, 1999; c) K. Haubennestel, P. Kramp (Byk-Chemie), EP 154678-B1, 1984; d) F. Campbell, F., Geary, J.M., Schofield, J. D. (ICI), USP 4,861,380, 1989.

[12] a) A.F. Johnson, M.A. Mohsin, Z.G. Meszena, P. Graves-Morris, J.M.S-Rev. Macromol. Chem. Phys., C39(3), 1999, 527, b) H. Fischer, J. Polym. Sci.:A. Polym. Chem., 1999, 37, 1885.

[13] H.L. Jakubauskas, J. Coat. Techn., 1986, 58(736), 71.

[14] a) O.W. Webster, W.R. Hertler, D.Y. Sogah, W.B. Farnham, T.V. RajanBabu, J. Am. Chem. Soc. 1983, 5706; b) D.Y. Sogah, W.R. Hertler, O.W. Webster, G.W. Cohen, Macromolecules, 1987, 20, 1473; c) US 4417034, 1982 (Du Pont).

[15] a) H.J. Spinelli, Prog. Org. Coat. 1996, 27, 255; b) US 4656226, 1985 (Du Pont); c) US 4755563, 1986 (Du Pont); d) US 4812517, 1987 (Du Pont); WO 99/03938, 1998 (Du Pont).

[16] a) K. Matyjaszewski, J. Xia, Chem. Rev. 2001, 101, 2921; b) WO 96/30421, 1996. (Carnegie-Mellon University).

[17] US 4581429, 1984 (CSIRO).

[18] a) WO 00/40630, 2000 (Ciba SC); b) WO 01/51533, 2001 (Ciba SC); c) WO 01/ 44376, 2001 (PPG); d) WO 01/44388, 2001 (PPG); e) WO 01/44389 2001 (PPG).

[19] M.O. Zink, A. Kramer, P. Nesvadba, Macromolecules 2000, 33, 8106; GB 2335190, 2000 (Ciba SC); GB 2342649, 2000, (Ciba SC). 